\title{
FLANN Detector Based Filtering of Images Corrupted by Impulse Noise
}

\author{
${ }^{1}$ Banshidhar Majhi and Mowafak Fathi \\ College of Computer Engineering and Information Technology \\ Al Hussein Bin Talal University, Ma' an, Jordan \\ ${ }^{1}$ On leave from National Institute of Technology, Rourkela, India
}

\begin{abstract}
We present a novel non-linear scheme for image restoration based on neuro-detector using Functional Link Artificial Neural Network (FLANN) followed by an improved spatial filter. The method is applied to images corrupted by impulse noise with varying strengths and different noise probability. The neural detector is based on the concept of training or learning by examples. When trained properly, the detector used to detect impulse noise in any image degraded by impulse noise. Hence, the method is suitable for real time image restoration applications. The simulated results obtained from the proposed scheme outperforms existing approaches are highly satisfactory and it outperforms the earlier suggested methods in terms of residual NSR in restored images.
\end{abstract}

Key words: Impulse Noise, Neural Network, Detection of Impulse Noise, Selective Filtering

\section{INTRODUCTION}

When images are acquired and processed, an original image may become degraded in various ways. Degradation may be in the form of random noise and interference, blur induced by camera mis-focus and motion, lens and film nonlinearity, impulse due to recording medium, and other mechanisms. Image restoration methods generally model the degradation process and apply an approximately inverse process to the degraded image to recover the original image [1]. The effectiveness of such image restoration techniques depends on the availability and completeness of knowledge about the degradation process as well as on the structure of the processing scheme implementing the restoration. Various image restoration methods have been proposed in the literature on digital image processing. Descriptions of image restoration techniques can be found in books on image processing such as [2]. Traditional image restoration methods are based on linear processing of image signals. Weiner filters [3] and recursive (Kalman) filters [4] fall under this category.

There are also nonlinear techniques for image restoration. These methods include maximumlikelihood [5] and maximum a posteriori (MAP) estimation technique [6]. Nonlinear methods based on modeling the degraded image, and hence called as model based methods.

However, very few works have been reported for impulse noise removal using nonlinear methods. A Double Derivative based impulse noise detection scheme is reported in [7]. Recently, the authors have proposed a fuzzy logic based impulse noise detection scheme [8], which performs better than the previous method [7] in terms of noise detection capability, edge retention, and noise removal. Another relatively new approach to model based image restoration uses Artificial Neural Network (ANN) filters. Hopfield type recurrent neural networks have been studied for restoration of images degraded by linear distortion $[9,10]$. ANN is also widely used for impulse noise detection and removal. When the impulse noise is of varying amplitude, a multi layer neural network is required. Such a work is reported in [11], which utilizes Radial Basis Function (RBF) network. In this paper, we propose a functional link neural network (FLANN) to detect the presence of an impulse of varying amplitude and if present, remove it by using improved spatial filtering. This scheme outweighs the earlier scheme proposed [11] in terms of noise rejection in $\mathrm{dB}$. To construct the neural detector, we use supervised learning based on back propagation. It is assumed that the image does not contain any sharp rise or fall in grayness at any of the pixel positions. The details of detection and filtering process of the proposed scheme are given

Proposed Scheme: A general discrete time model for image degradation can be expressed as:

$\mathrm{X}(\mathrm{m}, \mathrm{n})=\mathrm{Y}(\mathrm{m}, \mathrm{n})+(\mathrm{m}, \mathrm{n})$

where, $\mathrm{Y}(\mathrm{m}, \mathrm{n})$ represents the original image, $\mathrm{X}(\mathrm{m}, \mathrm{n})$ is the observed degraded image and $(\mathrm{m}, \mathrm{n})$ is the additive impulse noise of varying strengths added with a probability p. Since $\mathrm{p} \ll 1$, it is desirable to filter only those pixels which are corrupted by noise to minimize blurring in the restored image. To attain this objective we propose a noise detection scheme in the test pixel location, followed by an improved spatial filtering, if at all the test pixel is corrupted. The 
detection and filtration processes are described below in detail.

\section{Impulse Noise Detection}

1. Consider a $3 \times 3$ test window $\mathrm{X}_{\mathrm{T}}$ from $\mathrm{X}(\mathrm{m}, \mathrm{n})$ as:

$$
X_{T}=\left(\begin{array}{lll}
x_{i-1 j-1} & x_{i-1 j} & x_{i-1, j+1} \\
x_{i, j-1} & x_{i, j} & x_{i, j+1} \\
x_{i+1, j-1} & x_{i+1, j} & x_{i+1, j+1}
\end{array}\right)
$$

2. Compute ${ }_{i} s, i=1, \ldots, 4$ as follows:

$$
\begin{aligned}
& 1=\left(x_{i-1, j-1}+x_{i+1, j+1}\right) / 2-x_{i, j} \\
& 2=\left(x_{i+1, j-1}+x_{i-1, j+1}\right) / 2-x_{i, j} \\
& 3=\left(x_{i, j-1}+x_{i, j+1}\right) / 2-x_{i, j} \\
& 4=\left(x_{i-1, j}+x_{i+1, j}\right) / 2-x_{i, j}
\end{aligned}
$$

3. Pass all is through the FLANN detector which produces the output $\mathrm{O}_{i, j}$.

4. The fuzzy rules for the impulse detection in a test pixel $x_{i, j}$ are

* If $\mathrm{O}_{\mathrm{i}, \mathrm{j}}$ is high then the test pixel $\mathrm{x}_{\mathrm{i}, \mathrm{j}}$ is corrupted.

* If $\mathrm{O}_{i, j}$ is low then the test pixel $\mathrm{x}_{\mathbf{i}, j}$ is not corrupted.

To obtain a binary decision regarding the presence of the impulse, $D_{i, j}$ is passed through a hard limiter $(H)$ defined as:

$$
D_{i, j}=H\left(O_{i, j}\right)= \begin{cases}0, & O_{i, j}>T \\ 1, & \text { Otherwise }\end{cases}
$$

where, $\mathrm{T}$ is the threshold value.

If $D_{i, j}$ is zero, then the filtering process is carried out, otherwise the detection process is repeated for the next window. Figure 1 depicts the neural detector scheme.

Impulse Noise Filtration: Usually, the neighborhood of the corrupted pixel retains most of its intensity characteristics, when the probability of corruption is less. But this assumption becomes invalid, if the probability of corruption is very high. This is true as the neighbor pixels may also be corrupted and will contribute in the restoration of the corrupted pixel. As a result the restored images will be blurred. Hence it is desirable to test the healthiness of the neighbor pixels before calculating the new pixel intensity value for the corrupted pixel. We propose a similar filtering scheme. However, the pixel locations $x_{i-1, j-1}, x_{i-1, j}, x_{i-1, j+1}, x_{i, j-1}$ of the test window $X_{T}$ have already undergone the filtration process earlier, it is undesirable to test their healthiness. Hence, rest neighborhood pixels are tested through the neural detector. The corrupted pixel $x_{L, j}$ is a part of the test windows corresponding to rest neighborhood pixels for their healthiness testing.

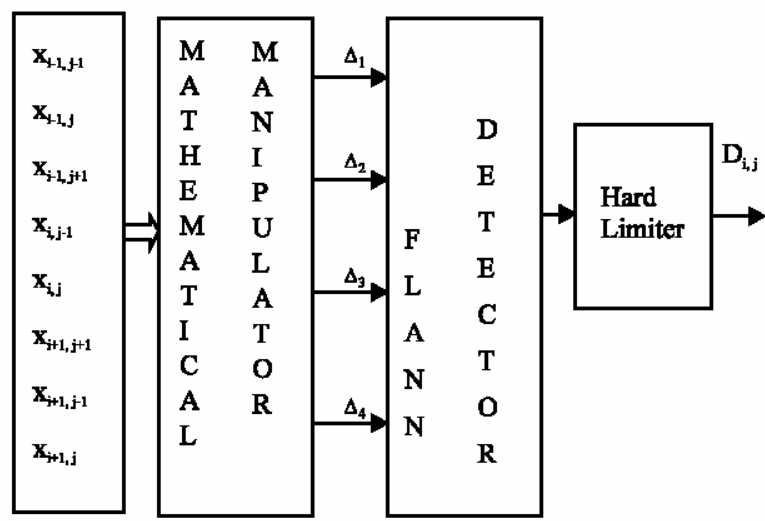

Fig. 1: The Proposed FLANN Detector

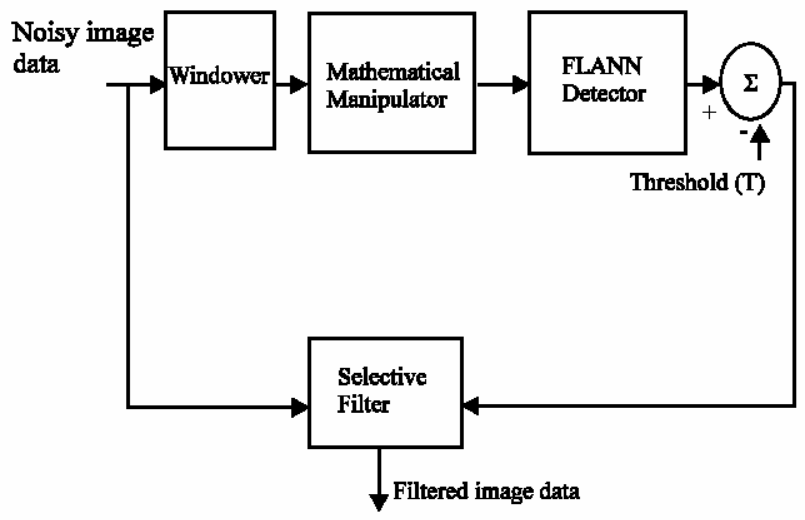

Fig. 2: Filter Structure

Hence, for more accuracy the pixel $x_{L, j}$ is replaced with the average of already filtered pixels. Finally, the average of all healthy pixels in the neighborhood is substituted for corrupted pixel location. The filtering process can be summarized as follows:

* Replace $x_{i, j}$ with $\left(x_{i-1, j-1}+x_{i-1, j}+x_{i-1, j+1}+x_{i, j-1}\right)$ 14

* Set $D_{i-1, j-1}, D_{i-1, j}, D_{i-1, j+1}, D_{i, j-1}$ each to 1 for the filtered locations.

* Pass the test windows corresponding to the pixels $\mathrm{x}$ $i, j+1, x_{i+1, j-1}, x_{i+1, j}$ and $x_{i+1, j+1}$ through the FLANN detector.

* Pass the output through the hard limiter to compute $D_{i, j+1}, D_{i+1, j-1}, D_{i+1, j}$ and $D_{i+1, j+1}$.

* Replace $\mathrm{x}_{\mathrm{I}, \mathrm{j}}$ by the average of the healthy pixels as:

$\mathrm{x}_{\mathrm{i}, \mathrm{j}}=\sum_{\mathrm{i}=1}^{3} \sum_{\mathrm{j}=1}^{3}\left(\mathrm{x}_{\mathrm{i}, \mathrm{j}} * \mathrm{D}_{\mathrm{i}, \mathrm{j}}\right) / \sum_{\mathrm{i}=1}^{3} \sum_{\mathrm{j}=1}^{3} \mathrm{D}_{\mathrm{i}, \mathrm{j}}$

The moving window $X_{T}$ is then shifted by one row and then by one column until the whole image is covered. Both detection and filtering process is repeated for all windows. The overall structure of the filtering scheme is depicted in Fig. 2. 
Table 1: Estimated Residual NSR of Different Restored Images

\begin{tabular}{|c|c|c|c|c|c|c|}
\hline \multirow[b]{2}{*}{$\%$ of noise } & \multicolumn{3}{|l|}{ LENA image } & \multicolumn{3}{|l|}{ LISA image } \\
\hline & $\begin{array}{l}\text { Noisy image } \\
\text { (NSR in dB) }\end{array}$ & $\begin{array}{l}\text { Restored image } \\
\text { (NSR in } \mathrm{dB})\end{array}$ & $\begin{array}{l}\text { Noise rejection } \\
\text { (in } \mathrm{dB} \text { ) }\end{array}$ & $\begin{array}{l}\text { Noisy image } \\
\text { (NSR in } \mathrm{dB} \text { ) }\end{array}$ & $\begin{array}{l}\text { Restored image } \\
\text { (NSR in } \mathrm{dB} \text { ) }\end{array}$ & $\begin{array}{l}\text { Noise rejection } \\
\text { (in } \mathrm{dB} \text { ) }\end{array}$ \\
\hline 5 & -13.8543 & -33.1850 & 19.3307 & -13.6314 & -41.0183 & 27.3869 \\
\hline 10 & -10.8738 & -33.1259 & 22.2521 & -10.7016 & -39.6304 & 28.9287 \\
\hline 15 & -9.1596 & -32.4265 & 23.2669 & -8.9967 & -36.9037 & 27.9070 \\
\hline 20 & -7.8893 & -31.2909 & 23.4016 & -7.7718 & -34.0056 & 26.2338 \\
\hline 25 & -6.8779 & -29.8346 & 22.9567 & -6.6931 & -32.7772 & 26.0842 \\
\hline 30 & -6.1295 & -28.2239 & 22.0944 & -5.9549 & -29.3743 & 23.4194 \\
\hline 35 & -5.4580 & -26.6046 & 21.1465 & -5.2299 & -26.7896 & 21.5596 \\
\hline 40 & -4.8855 & -23.9719 & 19.0864 & -4.7066 & -25.2861 & 20.5795 \\
\hline
\end{tabular}

Table 2: Comparative Residual NSR

\begin{tabular}{lll}
\hline Method & $\mathrm{p}=10 \%$ & $\mathrm{p}=20 \%$ \\
\hline Median filter & $-23.02 \mathrm{~dB}$ & $-22.22 \mathrm{~dB}$ \\
L-filter & $-23.76 \mathrm{~dB}$ & $-22.56 \mathrm{~dB}$ \\
Selective median & $-26.16 \mathrm{~dB}$ & - \\
SD-ROM & $-27.10 \mathrm{~dB}$ & $-23.82 \mathrm{~dB}$ \\
Combined system & $-30.68 \mathrm{~dB}$ & $-22.56 \mathrm{~dB}$ \\
Adaptive filtering [11] & $-31.20 \mathrm{~dB}$ & $-25.38 \mathrm{~dB}$ \\
New approach & $-33.13 \mathrm{~dB}$ & $-31.29 \mathrm{~dB}$ \\
\hline
\end{tabular}

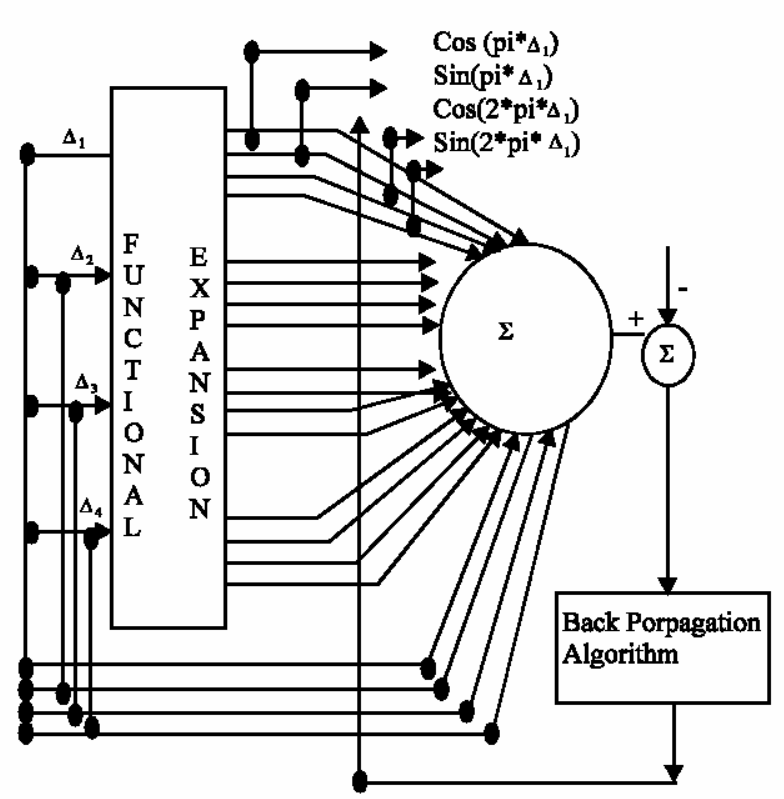

Fig. 3: FLANN Structure

Training Process: The neural network is trained with some known patterns of normalized LENA image corrupted by $10 \%$ impulse noise of varying strength lying between 0.3 to 0.7 . A total of four hundred patterns out of which two hundred noisy and two hundred non-noisy locations are selected at random for training. The value of ${ }_{\mathrm{I}} \mathrm{s}, \mathrm{i}=1 \ldots 4$ are calculated using the expression as mentioned in Eqn. [2]. The inputs to FLANN are I's and their functional expansion $\cos$ (PI $\left.*_{\mathrm{I}}\right), \cos \left(2 * \mathrm{PI} *_{\mathrm{I}}\right), \sin \left(\mathrm{PI} *{ }_{\mathrm{I}}\right)$, and $\sin \left(2 * \mathrm{PI} *{ }_{\mathrm{I}}\right)$. These expansions yield the enhanced nonlinear representation of the input patterns.

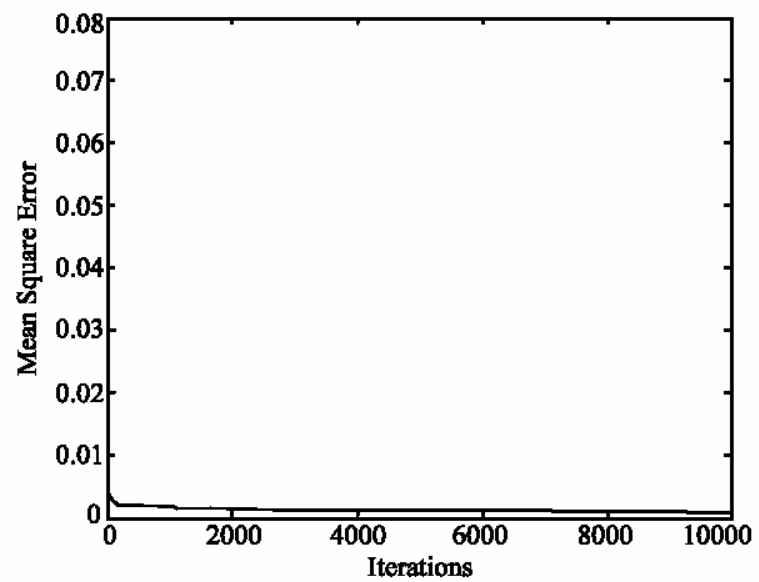

Fig. 4: Error Convergence Characteristics

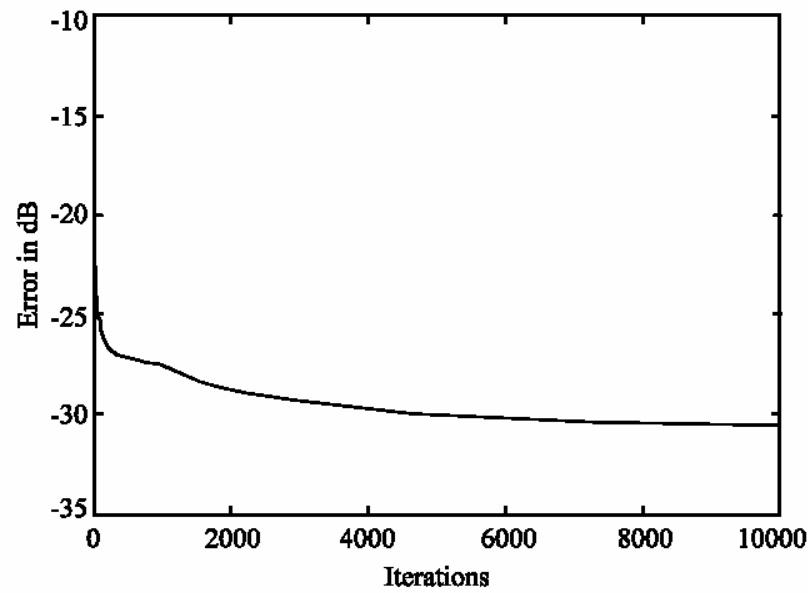

Fig. 5: Mean Square Error in dB

The target output corresponding to noisy pixel pattern is 0.9 and for non-noisy one it is chosen to be 0.1. This network provides large reduction in computational requirements and high convergence speed. The training is conducted using the normal back propagation algorithm. The network structure is shown in Fig. 3. It can be seen from Fig. 4 that the error rate converges to $10^{3}$ in 10,000 iterations. The mean square error in $\mathrm{dB}$ for the learning process is shown in Fig. 5. 


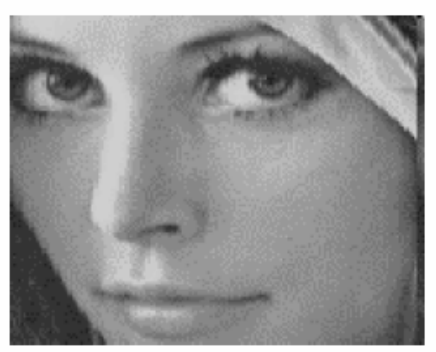

original image

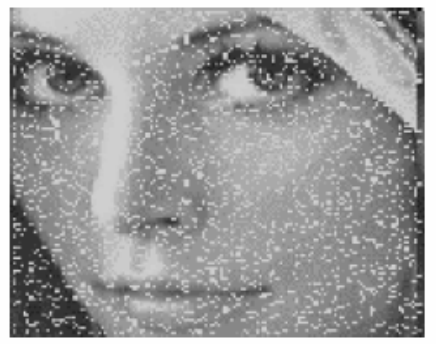

Corrupted image $(15 \%)$

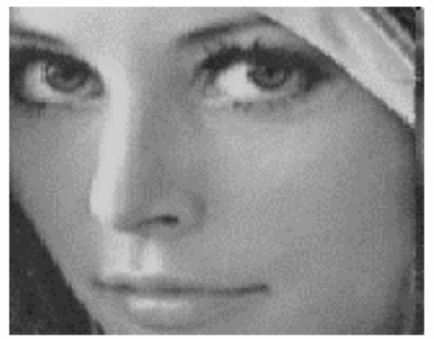

Restored image

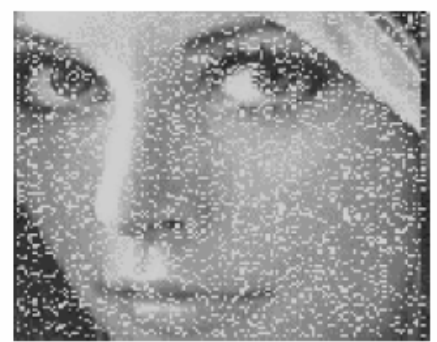

Corrupted image $(20 \%)$

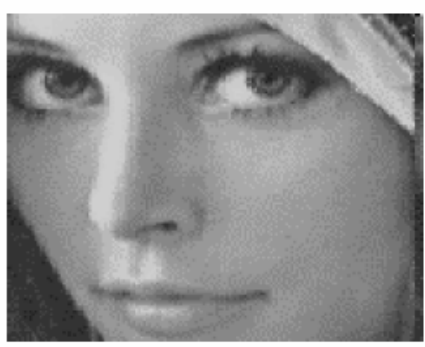

Restored image

(a) LENA image

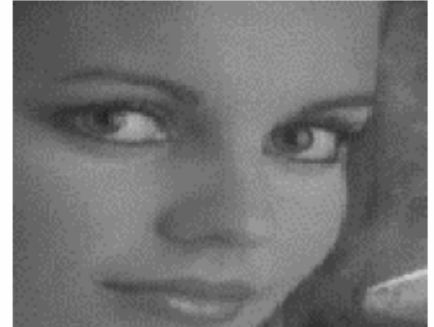

original image
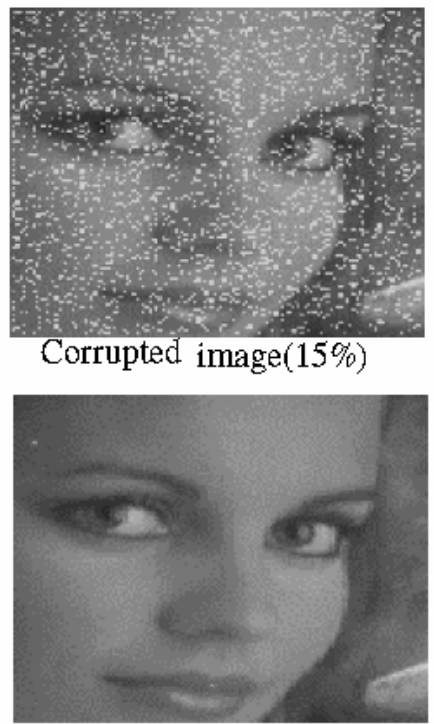

Restored image

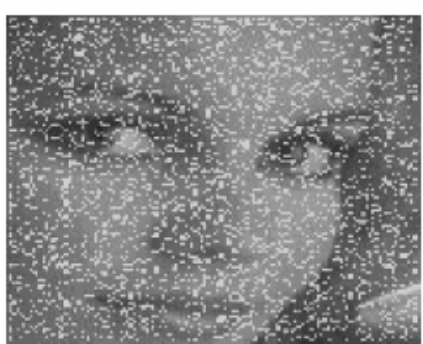

Corrupted image(20\%)

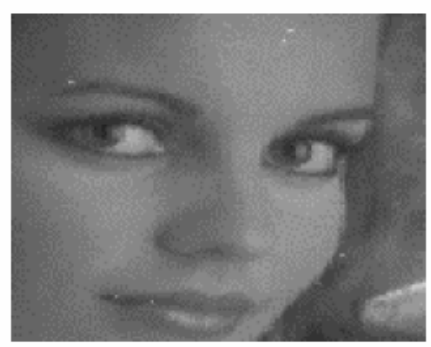

Restored image

(b) Lisa image

Fig. 6: Restored Results by the Proposed Scheme 


\section{SIMULATION AND RESULTS}

In order to visualize the difference in performance between standard nonlinear filtering [11] and our new approach, the proposed scheme is simulated using two standard images LENA and LISA. Probability of corruption in the images is varied between 5 to $40 \%$. The threshold value $\mathrm{T}$ chosen for the simulation is 0.42 . The restored results for the LENA and LISA images are shown in Fig. 6. The neural detector trained for LENA image is used to detect the impulse noise from LISA image and the noise detection capability is observed to be highly satisfactory. The noise to signal ratio (NSR) in $\mathrm{dB}$ is estimated using conventional formula and shown in Table 1. The residual NSR associated with the restored images are used as the index of efficiency of our proposed adaptive method. The results obtained were compared to that with method suggested [11]. The comparative performance is shown in Table 2.

\section{CONCLUSION}

This study proposes a novel and efficient neural detection with improved spatial filtering method of removing impulse noise contaminated with 2-D image. The method involves two steps: impulse detection and spatial filtering. The simulation study has been performed on two standard images. The performance study of the proposed technique has been made in terms of residual NSR in dB. In general, it is observed that the proposed technique yields lower NSR in restored images to the tune of $22 \mathrm{~dB}$ and $26 \mathrm{~dB}$ in LENA and LISA images respectively. Thus, it can be concluded that the method possesses better noise detection and filtering capability.

The unique feature of the proposed technique is that it does not involve all the pixels for filtering. The corrupted pixels are identified and those pixels only undergo spatial filtering there by, the computational complexity is greatly reduced.

In addition, the neural detector trained in one image pattern is also applicable to other similar images. Hence it can be applicable for real time image restoration.

\section{REFERENCES}

1. Cha, I. and S.A. Kassam, 1996. RBFN restoration of nonlinearly degraded images. IEEE Trans. on Image Processing. 5: 964-975.

2. Jain, A.K., 1990. Fundamentals of Digital Image Processing. Englewood Cliffs, NJ: PHI.

3. Chellapa, R. and R.L. Kashyap, 1982. Digital image restoration using spatial interactive models. IEEE Trans. on Accoust., Speech, Signal Processing, ASSP-30, pp: 461-472.

4. Dikshit, S.S., 1982. Arecursive Kalman window appraoch to image restoration. IEEE Trans. on Accoust., Speech, Signal Processing, ASSP-30, pp: 125-129.

5. Jeng, F. and J. Woods, 1991. Compound GaussMarkov random fields for image restoration. IEEE Trans. on Signal Processing. 39: 683-697.

6. Bouman, C. and K. Sauer, 1993. A generalised Gaussian image model foe edge-preserving MAP estimation. IEEE Trans. on Image Processing, 2: 296-310.

7. Panda, G. and S.K. Maharatha, 1996. Efficient filtering of Image data corrupted by impulse noise. Proc. of Intl. Conf. on Signal Processing Applications and Technology (ICSPAT'96) Boston, 1: 1032-1035.

8. Panda, G., B. Majhi and P.K. Dash, 1999. Fuzzy logic based Impulse Noise detection and restoration of image. Proceedings of Intl. Conf. on Advance Computing, University of Roorkee, pp: 812, Dec. 20-22.

9. Zhou, Y., R. Chellapa, A. Vaid and B.K. Enkins, 1991. Image restoration using a neural network. IEEE Trans. on Accoust., Speech, Signal Processing, 36: 197-209.

10. Paik, J.K. and A.K. Katasaggelos, 1992. Image restoration using Hopfield network. IEEE Trans. on Image Processing, 1: 49-63.

11. Sucher, R., 1995. A new adaptive nonlinear filtering algorithm. Proc. of IEEE Intl. Conf. on Signal Processing, Circuits and Systems, pp: 1-4, Singapore. 